\title{
Classification of Electrocardiogram Cardiac Arrhythmia Signals Using Genetic Algorithm - Support Vector Machines
}

\author{
M Ramkumar', M Mathankumar ${ }^{2}$ and A. Manjunathan ${ }^{3}$ \\ ${ }^{1}$ Electronics and Communication Engineering, Sri Krishna College of Engineering and Technology, Coimbatore \\ ${ }^{2}$ Electrical and Electronics Engineering, Kumaraguru College of Technology, Coimbatore \\ ${ }^{3}$ Electronics and Communication Engineering, K Ramakrishnan College of Technology, Trichy
}

\section{ABSTRACT}

This research study has been focused on exploring the novel approach for classifying the arrhythmia disease of cardiac muscle. The proposed methodology determines the combination of Genetic Algorithm and Support Vector Machines techniques. Initially, the feature extraction of twenty-four features has been made from the ECG waveform. The acquisition of these features has been made by partial automatic extraction from the amplitude (voltage)-time parameters of $P$ peak, Q peak, R peak, S peak and T peak feature sets of an ECG waveform. Genetic Algorithm is utilized for improving the performance of generalization in the Support Vector Machine classifier. In order to proceed with this task, the optimization of Support Vector Machine classifier is done by performing the search of the parameter with the best value which makes tuning of its discriminate function and seeking for the best feature subset with which in response does the optimization of the fitness function associated with the classification results. Certain simulations have been carried out with the help of MATLAB software with which the results over the experiments determines the demonstration that the proposed methodology does the best classification of ECG waveforms in detecting the cardiac arrhythmias. The recorded ECG dataset has been acquired from MIT-BIH arrhythmia database and 4 different sorts of arrhythmias has been considered for performing the classification task and it is obtained with the results of 97.45\% of accuracy, 95.2\% of sensitivity, $95.4 \%$ of specificity and $96.23 \%$ of positive predictivity.

KEY WORDS: CARDIAC ARRHYTHMIA, ECG WAVEFORM, ECG CLASSIFICATION, FEATURE EXTRACTION, GENETIC ALGORITHM, SUPPORT VECTOR MACHINE.

\section{INTRODUCTION}

Classifying the ECG (Electrocardiogram) signals into various categories of arrhythmia disease is the tedious task of recognizing the patterns. However, ECG signal analysis is one of the most efficient methods available for making the diagnosis of cardiac heart arrhythmias.

\section{ARTICLE INFORMATION}

*Corresponding Author: mathankumarbit@gmail.com Received 11th Oct 2020 Accepted after revision 13th Dec 2020 Print ISSN: 0974-6455 Online ISSN: 2321-4007 CODEN: BBRCBA

Thomson Reuters ISI Web of Science Clarivate Analytics USA and Crossref Indexed Journal

\section{Clarivate
Analytics}

NAAS Journal Score 2020 (4.31) SJIF: 2020 (7.728)

A Society of Science and Nature Publication,

Bhopal India 2020. All rights reserved.

Online Contents Available at: http//www.bbrc.in/

Doi: http://dx.doi.org/10.21786/bbrc/13.11/29
ECG arrhythmia Classification on the basis of computer approach could provide accuracy on higher rate of degree and would offer a mass potential in the screening of heart abnormalities. Achievement in doing the cardiac arrhythmia classification successfully is done by determining the ECG characteristic shape that makes the effective discrimination between the required categories of diagnosis. Certain conventional methods for the identification of typical heart beat from the ECG wave component are determined by few parameters such as area, duration and amplitude. Various statistical and morphological features are considered for inhibiting the training and testing of ECG parameters in terms of vectors to do the process of classification. Few are made on the basis of experiments done in the laboratory whereas others are made with the involvement of symptoms identified clinically.

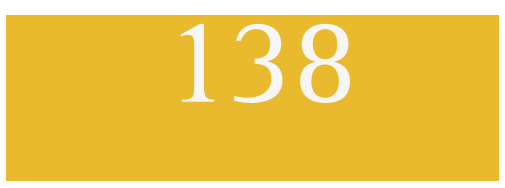


In this proposed study, the ECG signals are acquired from MIT-BIH cardiac arrhythmia database. Many researchers have been widely utilizing this database for testing their different algorithms in detecting the cardiac arrhythmia and do the process of classification. The proposal of certain methods has been made for the ECG cardiac arrhythmia classification. The technique that has been presented in first study is on the basis of Fisher Linear discriminant (Acharya U R et al., 2008). The duration of R-R interval and the spatial distance inbetween the existence of $\mathrm{P}$ peak and T peak waveforms has been perceived. On utilizing the mentioned features, the application of Fisher's Linear Discriminant has been made. The technique on the Support Vector Machine (SVM) for detecting Premature Ventricular Contraction arrhythmia is declared as more efficient algorithm when compared with ANFIS (Osowski, S, T.H. Linh, 2001). Later on, the proposal over the selection of features and the cardiac arrhythmia classification on the basis of Particle Swarm Optimization-Support Vector Machine (PSOSVM) has been made (Chazal P., et al., 2004).

A novel approach on the basis of fuzzy-neural network has been described for classifying the ECG cardiac rhythms (Bandyopadhyay, S, S.K. Pal, 2007). In this classification mechanism, the characterization of QRS complex for the ECG waveform is determined by the polynomials of Hermite, with which the fuzzy-neural classifier is being fed by its coefficients. The proposal of Cardiac Arrhythmia detection with respect to Wavelet Transform (WT) and Independent Component Analysis (ICA) for extracting essential features has been made (Mark, R.G., et al., 1997). Later on, the proposal for classifying ECG cardiac arrhythmia by utilizing the ECG beats of huge dataset is carried out in possessing the training of neural network using the features of timing and wavelet. The identification of authors has been made in such a way that the 4th scale of dyadic WT with the wavelet of quadratic spline combined together with the post/pre interval ratio of R-R is considered as very efficient technique in differentiating and categorizing the PVC (Premature Ventricular Contraction) and Normal beats from the alternate class of ECG beats.

Even though, the development of huge techniques in classifying the ECG arrhythmias or the ECG waveforms is being carried out by various researchers, this study has also been proposed in one such way of classifying the cardiac arrhythmia by utilizing the hybrid technique of Genetic Algorithm and Support Vector Machines. The structure of this paper has been made as follows. The second section makes the description of classification methodology by Support Vector Machines (SVM). The third section determines the description of proposed technique for extracting the features in terms of feature extraction. The fourth section makes the description of the dimensionality (features) reduction. The proposed Genetic Algorithm-Support Vector Machine (GA-SVM) technique of classification has been described in fifth section. Lastly the sixth and seventh section makes the presentation over conclusion and future works to be carried on respectively.

\section{MATERIAL AND METHODS}

Classification using Support Vector Machine (SVM): In this particular section, the brief explanation and the review of 2 class SVM and the multi class Support Vector Machine classification technique has been made. Support Vector Machine (SVM) (Clifford, G.D., et al., 2006) is considered to be as very powerful and popular technique in the learning that has been created for patterns since its characteristics over the support of multi or high dimensional information and also due to the yield of best properties over generalization. Moreover, Support Vector Machines have many applications over recognizing the patterns, applications over data mining such as categorizing the text (Acharya U R et al., 2017, Homaeinezhad, M.R., et al., 2011), recognition of phoneme (Banerjee, S., Mitra, M., 2014), detection of threedimensional object (Liu, T., et al., 2016), classification of images (Mitra, M., Samanta, R.K., 2013) and the field of bio-informatics (Martis, R.J., et al., 2013b). At the initial stage, the formulation of Support Vector Machine has been made for the problems of classifying binary (2-class). The extension over this particular technique has been made to the problems of multi-level class which might neither be straightforward nor be the isolated one. DAG-SVM is one of the techniques with which the proposal has been made for the extension of Support Vector Machine Classifier for supporting the classification of multi-class domain (Thomas, M., et al., 2015).

\section{Formulation of Binary Support Vector Machine}

Let $X=\left\{\left(x_{i}, y_{i}\right)\right\}_{i=1}^{n}$

be considered as the set of training samples $\mathrm{n}$, with which $x_{i} \in \mathcal{R}^{m}$ is the sample of $\mathrm{m}$-dimension in the space of input and $y_{i} \in\{-1,1\}$ is denoted as the label class for the sample $\mathrm{x}_{\mathrm{i}}$. Support Vector Machine determines the finding over the optimal separating hyperplane (OSH) with the minimal errors resulted from the classification. The hyperplane separation in the linear form is being represented as in equation (1).

$$
f(x)=w^{T} x+b
$$

Where $\mathrm{w}$ denotes the vector of weight and $\mathrm{b}$ denotes the bias respectively. The hyperplane with optimal characteristics could be acquired by resolving the problem of optimization, where $\xi_{\mathrm{i}}$ is determined as the variable of slack for acquiring the soft margin whereas the $\mathrm{C}$ variable determines the control for the variables of slack. The value for the margin of separation enhances by providing the decrement for the $\mathrm{C}$ value.

In the SVM, a hyperplane which is optimum in nature has been acquired by enhancing the capability of generalization of Support Vector Machine by itself. However, if the data associated with the training are not separable linearly, the acquired classifier might not possess high ability of generalization although optimal determination of the hyperplanes are being carried out. For enhancing the separability in the linear fashion, the 
mapping for the original space of input has been made with the space of the dot product with high dimension represented as the feature space. Now utilizing the function of vector which is non-linear $\varphi(x)=\left(\varphi_{1}(x), \ldots \ldots \varphi_{1}(x)\right)^{T}$ with which the mapping is made over the input vector $\mathrm{x}$ with $\mathrm{m}$-dimension into the feature space of l-dimension, the OSH (Optimum Separation Hyperplane) in the feature vector space is denoted by equation (2).

$$
f(x)=w^{T} \varphi(x)+b
$$

The decision function that has been designated for the test information is given by equation (3).

$$
\begin{gathered}
D(x)=\operatorname{sign}\left(w^{T} \varphi(x)+b\right. \\
\text { Minimize } \frac{1}{2}\|w\|^{2}+C \sum_{i=1}^{n} \xi_{i} \\
\text { subject to } y_{i}\left(w^{T} \varphi\left(x_{i}\right)+b\right) \geq 1-\xi_{i} \\
\xi_{i} \geq 0, i=1, \ldots \ldots, n
\end{gathered}
$$

Figure 1: Functional Block Diagram of proposed classification of ECG arrhythmia

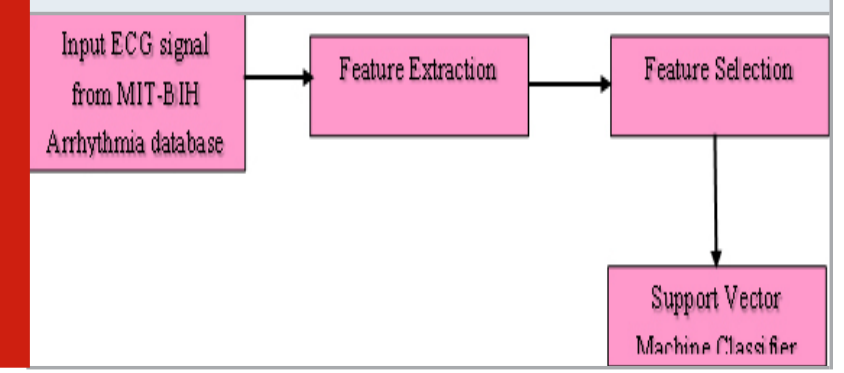

Figure 2: Representation of sample signal a) Normal Sinus Rhythm b) Left Bundle Branch Block Beat c) Premature Ventricular Contraction d) Right Bundle Branch Block Beat

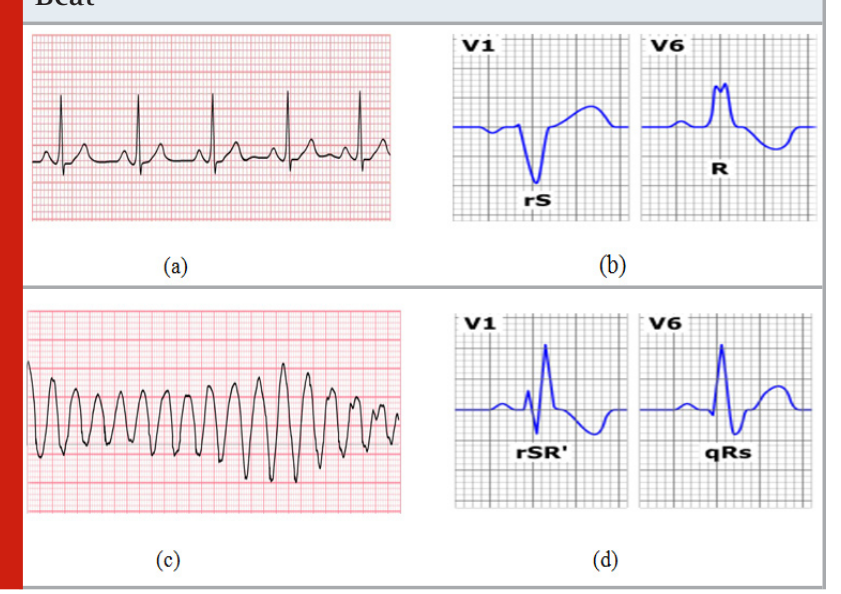

Support Vector Machine with Multi-Class: As the description made earlier Support Vector Machines are the classifiers with binary intrinsic characteristics. However, the ECG waveform classification makes the involvement for more than dual classes. For facing this particular problem, an adaptation for the total number of strategies involved under the classification of multiclass has been made [15] Daubechies, I., 1998). Among those strategies, the most essential strategies are one-againstall (OAA) strategies and one-against-one (OAO) strategy. The construction for the One Against One (OAO) has been made with $(n(n-1)) / 2$ decision functions for most of the classification pair combinations. The results over the experimentation has indicated that the One-Against-One is best sustainable and suitable for the practical usage. In this proposed study, the process of OAO has been utilized for the multi class classification of ECG signal (Takeuchi, K., Collier, N., 2003).

Methodology for the Extraction and Selection of features: In this particular section, the discussion has been made in such a way that the characteristics of the ECG features that has been extracted and the design procedure for extracting the ECG features has been explained. Figure 1 depicts the functional block diagram of the proposed classification mechanism of ECG arrhythmia. The flowchart for the algorithm execution has been discussed in the following chapter.

Description of Dataset: The conduction of the experiments has been made over the ECG waveform as the raw base signal which is being taken for signal classification. In most of the research studies the realization over the ECG signal classification has been acquired from MIT-BIH cardiac arrhythmia database (Wang, T.-Y., Chiang, H.-M., 2007) from the physionet and it is available online with open access. It has been widely utilized for evaluating the performance of various classifiers. The database is totally consisting of 48 recordings with which each individual recording is being made with 30 minutes of time duration. The recording of each data is made is made in dual channels and it is denoted in terms of second modified limb lead and sixth modified limb lead.

Particularly, the type of ECG beat which has been considered in our proposed study is being determined by the following classes. They are Normal Sinus Rhythm (NSR), Left Bundle Branch Block Beat (LBBB), Premature Ventricular Contraction (PVC) and Right Bundle Branch Block Beat (LBBB). The sample representations of those ECG beat signals has been depicted in figure 2. The selection of those beats is made from 101, 104, 106, 109, 118, 124, 207, 214, 219, 221, 231 and 234 as shown table 1 .

Reduction of Noise: In the initial stage of extracting the features, the performance over the wavelet transform has been made in order to enhance the reduction of noises. The WT (Wavelet Transform) permits the non-stationary signal processing such as Electrocardiogram signal. The representation of raw ECG signal and the Filtered ECG signal has been depicted in figure 3 and figure 4 respectively. 
Description of Features: For each of the ECG signal, totally 19 features which are temporal in its characteristics such as interval of R-R, interval of $\mathrm{P}-\mathrm{Q}$, interval of $\mathrm{P}-\mathrm{R}$, interval of $\mathrm{P}-\mathrm{T}$ and added to that the recognition of 3 morphological features has been made. Manually, the extraction of these features has been made for each ECG beat and stored into an isolated vector. The tag of each vector has been made with one of its 4 labels like NSR, PVC, LBBB and RBBB respectively.

\begin{tabular}{l|c|c|c|}
\multicolumn{4}{|c|}{ Table 1. Description of Dataset and Usage of numbers in simulation } \\
\hline $\begin{array}{l}\text { Class } \\
\text { Number }\end{array}$ & $\begin{array}{c}\text { Example of } \\
\text { record acquired } \\
\text { from MIT-BIH }\end{array}$ & $\begin{array}{c}\text { Total count } \\
\text { of ECG } \\
\text { beats used }\end{array}$ & Type of Beat \\
\hline 1 & 101,104 & 245 & Normal Sinus Rhythm (NSR) \\
\hline 2 & 106,109 & 108 & Premature Ventricular Contraction (PVC) \\
\hline 3 & 207,214 & 602 & Left Bundle Branch Block Beat (LBBB) \\
\hline 4 & 118,124 & 448 & Right Bundle Branch Block Beat (RBBB) \\
\hline
\end{tabular}

Figure 3: Raw acquired ECG signal

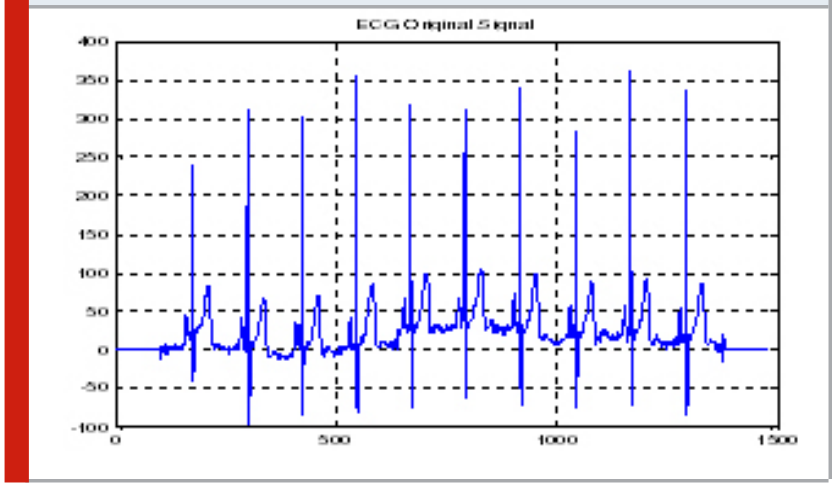

The extraction of the features has been made inclusive of the $\mathrm{P}$ peak, $\mathrm{Q}$ peak, $\mathrm{R}$ peak, $\mathrm{S}$ peak and $\mathrm{T}$ peak along with the time interval of R-R, S-T and P-T and interval of time over each of five various features from the preceding feature such as R-S, S-T and Q-R as denoted in figure 5 and also it is inclusive of the feature's voltage difference that has been denoted in terms of $E(Q)-E(S)$. Consideration of one more feature has been made in terms of voltage and time of R-R. The features which has been described is being represented in the following table 2. $\mathrm{Y}(\mathrm{R})$ denotes the $\mathrm{R}$ position of ECG wave component and W(R) denotes the position's value in that ECG component.

The computation of the minimum and the maximum values of ECG beats in the signal has been yielded with the formation of 3 features which are morphological in its characteristics. Scaling over each beat in the ECG signal has been made by utilizing the subsequent formula with which its range of each individual signal relies between 0 and 1 .

$$
h(t)=\frac{h(t)-\min (t)}{\max (t)-\min (t)}
$$

Figure 4: Filtered ECG signal

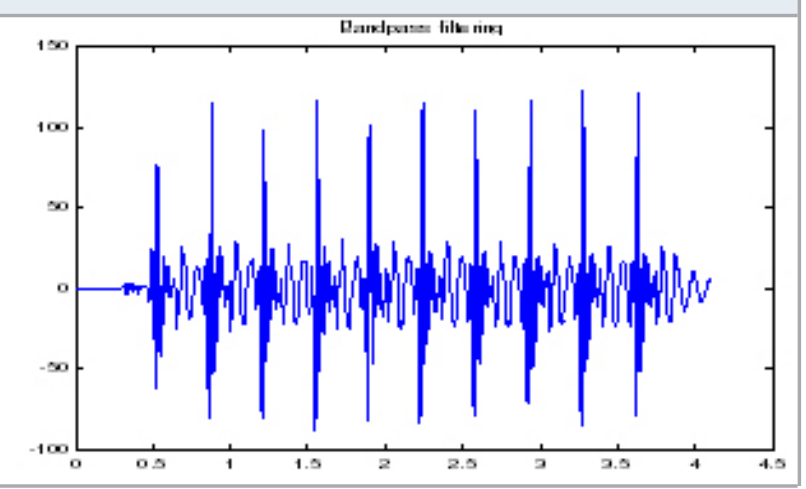

Figure 5: Representation of Features for ECG Wave Component

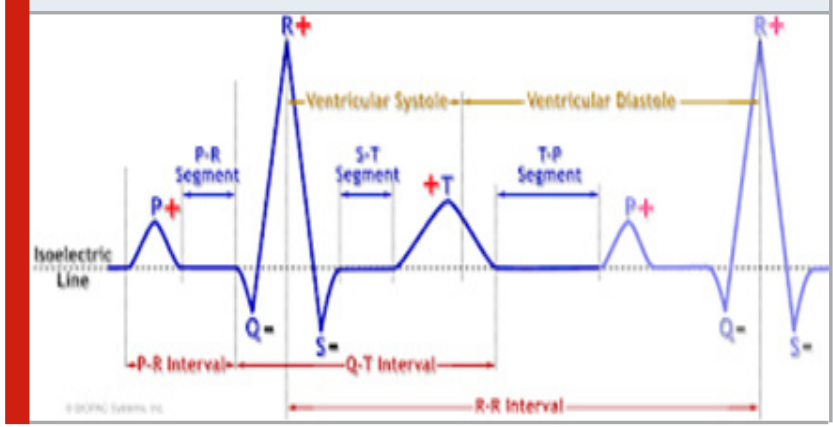

The maximum voltage and the minimum voltage in between the initial and the subsequent feature of $R$ is being computerized and followed with the performance of normalization process [0 1]. As determined earlier, the consideration over the percentage has been meant with the value which is larger than $0.2,0.5$ and 0.8 as 3 various features. Out of 22 features, 6 features are being represented as the basic features and they are as follows: $\mathrm{R} 1, \mathrm{R} 2, \mathrm{P}, \mathrm{Q}, \mathrm{T}$ and $\mathrm{S}$ and the remaining are represented as the features of derivatives. The calculations of the features that has been derived are made utilizing the basic features through the procedure of semi automation. The 
suggestion for the initial and the subsequent point of $R$ to the expert is being made by utilizing the minimummaximum based algorithm. Then the differentiation made by the experts for the appropriate points has been made in terms of $\mathrm{P}, \mathrm{Q}, \mathrm{R}, \mathrm{S}$ and $\mathrm{T}$.

Reduction of Features: Many researches in the area of selection of features and data analysis determines the suggestion that not every feature is being utilized for classification process (Bandyopadhyay, S, S.K. Pal, 2007, Mark, R.G., et al., 1997, Osowski, S, T.H. Linh, 2001). As on the case of contrary, few features might function as noisy parameters and hence it results with the reduction in the accuracy of classification. In this proposed study, two various approaches on the reduction of features has been accomplished. This proposed mechanism has shown that the technique on the basis of meta-heuristic has yielded best performance for the arrhythmia classification on ECG signal when compared to the method of statistical analysis (Bandyopadhyay, S, S.K. Pal, 2007).

Table 2. Description of features that has been utilized in the simulation

\begin{tabular}{|l|c|c|c|}
\hline $\begin{array}{l}\text { Feature } \\
\text { Number }\end{array}$ & $\begin{array}{c}\text { Description } \\
\text { of Features }\end{array}$ & $\begin{array}{c}\text { Feature } \\
\text { Number }\end{array}$ & $\begin{array}{c}\text { Description } \\
\text { of Features }\end{array}$ \\
\hline 1 & $\mathrm{Y}(\mathrm{R} 1)$ & 11 & $\mathrm{Y}(\mathrm{R} 2)$ \\
\hline 2 & $\mathrm{~W}(\mathrm{R} 1)$ & 12 & $\mathrm{~W}(\mathrm{R} 2)$ \\
\hline 3 & $\mathrm{Y}(\mathrm{S})$ & 13 & $\mathrm{Y}(\mathrm{R} 2)-\mathrm{Y}(\mathrm{R} 1)$ \\
\hline 4 & $\mathrm{~W}(\mathrm{~S})$ & 14 & $\mathrm{~W}(\mathrm{R} 2)-\mathrm{W}(\mathrm{R} 1)$ \\
\hline 5 & $\mathrm{Y}(\mathrm{T})$ & 15 & $\mathrm{Y}(\mathrm{S})-\mathrm{Y}(\mathrm{R} 1)$ \\
\hline 6 & $\mathrm{~W}(\mathrm{~T})$ & 16 & $\mathrm{Y}(\mathrm{T})-\mathrm{Y}(\mathrm{S})$ \\
\hline 7 & $\mathrm{Y}(\mathrm{P})$ & 17 & $\mathrm{Y}(\mathrm{P})-\mathrm{Y}(\mathrm{T})$ \\
\hline 8 & $\mathrm{~W}(\mathrm{P})$ & 18 & $\mathrm{Y}(\mathrm{Q})-\mathrm{Y}(\mathrm{P})$ \\
\hline 9 & $\mathrm{Y}(\mathrm{Q})$ & 19 & $\mathrm{Y}(\mathrm{R} 2)-\mathrm{Y}(\mathrm{Q})$ \\
\hline 10 & $\mathrm{~W}(\mathrm{Q})$ & & \\
\hline
\end{tabular}

Principal Component Analysis: Principal Component Analysis is determined as the probabilistic technique for making the reduction in the data dimension (Wang, T.-Y., Chiang, H.-M., 2007). It establishes the selection on the variable set which are not correlated with one another and parallelly each variable is declared as the linear combination of originated variables. The derivatives of the principal components are made from the original information such that the initial principal component calculates for the maximum proportionality of the original informational set variance inclusive of orthogonal components which are subsequently present that has also been accounted for the peak proportionality for the balance variance. The successive steps for the execution of Principal Component Analysis has been mentioned as follows. a. Determine the computation for the information mean vector

b. Determine the computation for the information covariance matrix

c. Determine the computation for the eigen matrix and the eigen value of the matrix of covariance

d. Formulation of the components utilizing the covariance matrix eigen vectors as the coefficients of weights.

It is sustained to be advertised that the classifier of the Principal Component Analysis (PCA) establishes its performance well for the entire informational datasets rather the evaluation of the performance could not be attained well for few groups of informational sets (Bandyopadhyay, S, S.K. Pal, 2007). Very few research studies (Ahmad, A.M., et al., 2013, Chang, C.C., Lin, C.J. 2011, Kressel, U.H.-G, 1999, Wang, T.-Y., Chiang, H.-M., 2007) has shown that the Principal Component Analysis (PCA) is not the standard technique for making the analysis over the non-linear information. It seems to be that the noisy information existence, abnormal range of features for which some are ranging between $\left[\begin{array}{ll}0 & 1\end{array}\right]$ whereas the other features are ranging between [0 10000], minimum variance of few essential features are the major reasons for the minimal performance of Principal Component Analysis. Table 5 determines the result over experimentation by Principal Component Analysis.

Genetic Algorithm: Genetic Algorithm is one of the optimized techniques that is being utilized for the reduction of features. Few alternate techniques of optimization with meta-heuristic characteristics like simulated annealing tabu search and the strategies of evolution are also declared as the candidates for enhancing this purpose. The demonstration over Genetic Algorithm has been made for converging into the partially optimized solution for many difficult and diverse problems as the stochastic and the powerful tools on the basis of natural evolution principle (Ebrahimzadeh, A. Khazaee, 2010). In most of the applications it is being utilized for the dimensionality reduction of features and the weighting of features (Vaseghi, S.V., 2008). The steps for the algorithm in executing GA has been mentioned as follows in Table 3.

The initial step in any of the genetic algorithm is to make the definition of the encoding process for allowing the description of a potential solution as a part of numerical vector and for making an attempt to make the randomized generation of population. The brief descriptions on the operators of GA has been made as follows.

a. Selection: The process of selection directly picks the individuals from the existing population on the basis of 
fitness parameters from each chromosome (Hsu, C.W. and C.-J. Lin, 2002).

b. Recombination: The main responsibility of the operation of crossover is to enable the creation of current individuals from the previous ones. Crossover is most probably determined as the statistical process which makes the exchange of data between few individuals of parent for making the generation of new individuals as child.

c. Mutation: The application of mutation is being done to single individual and makes the production of modified child mutant.

d. Function of Fitness: The main responsibility of the fitness function is to make the measurement of the solution's quality.

Genetic Algorithm-Support Vector Machine: In this particular section, the description over the Genetic
Algorithm-Support Vector Machine for classifying the cardiac arrhythmias is being made. In this system, the main aim is to make the selection process for the feature subset automatically in order to optimize the classifier of Support Vector Machine. The procedure has been described as follows in figure 6 .

Set up of Genetic Algorithm: The initial step in the genetic algorithm is to make the definition for the procedure of encoding which permits the description of any valid or strengthened solution as the vector of numerical value. The length of the vector has been chosen with the value of 23 with each individual component either saturates with the value of 1 or 0 which has been selected for the process of encoding. The synonym for the component that possess zero value is that the omission of the subsequent feature is being made and the synonym for the component that possess one value is vice versa. In the following experimentations, the original population is possessed with fifty chromosomes which has been selected randomly.

\begin{tabular}{|c|c|c|}
\hline & Algorithm Steps & Genetic Algorithm Description \\
\hline \multirow{9}{*}{$\begin{array}{l}\text { Input: Training } \\
\text { Information } \\
\text { Output: } \\
\text { Required Features }\end{array}$} & 0th Step & $\begin{array}{l}\text { Initialization of parameters (e.g. Size of population, } \\
\text { rate of crossover, rate of mutation and the } \\
\text { maximum count of population generation). }\end{array}$ \\
\hline & 1st step & Random Creation of initial population $(\mathrm{P}(0))$. \\
\hline & 2nd step & $\begin{array}{l}\text { Evaluation of current population (Computation } \\
\text { of fitness for entire chromosomes) }\end{array}$ \\
\hline & 3rd step & $\begin{array}{l}\text { While (satisfaction of termination condition is not being } \\
\text { made, step } 4 \text { to } 8 \text { has to be proceeded. }\end{array}$ \\
\hline & 4th step & Performance of selection over $\mathrm{P}(\mathrm{t})$ from $\mathrm{P}(\mathrm{t}+1)$ has to be made. \\
\hline & 5th step & $\begin{array}{l}\text { Performance of mutation and crossover has to be made from } \\
\text { the recombination of } \mathrm{P}(\mathrm{t})\end{array}$ \\
\hline & 6th step & $\begin{array}{l}\text { Evaluation of current population has to be made } \\
\text { in order to establish the computation of all fitness chromosomes. }\end{array}$ \\
\hline & \multirow{2}{*}{ 7th step } & Proceed with $t=t+1$ \\
\hline & & To be proceeded with step 3 \\
\hline
\end{tabular}

The process of swapping and the selection of roulette wheel has been utilized for the mutations and the crossover operations respectively. The operation of swap changes the position of dual samples randomly. The probability mutation parameter has to be selected with the value of 0.1 . The selection for the fitness function choice is very essential since it is on the basis of evaluation made by genetic algorithm, the best of solution has been given to each candidate for establishing the design of Support Vector Machine system of classification. In this proposed study the exploration for the correction rate in performing the classification of ECG signal is being made.
Classification of SVM with GA: The description of the procedure for the classification system on the basis of Support Vector Machine is as follows.

Step 1: Generation of initial random population of determined size of 50

Step 2: For the population of each individual chromosomes, perform the training of

$\frac{n(n-1)}{2}$ Support Vector Machine Classifiers.

Step 3: Utilizing the multiclass SVM technique (OAO) the computation for the fitness of each individual 
chromosomes is being made (feature subset).

Step 4: Selection of few individuals directly has to be made from the existing population on the basis of fitness values and make the regeneration of upcoming individuals from the previous ones.

Step 5: If the maximum count of iteration is not attained, then it has to be proceeded from 2 nd step.

Step 6: Selection of chromosomes with the best values of fitness has been made as the desired feature subset.

Step 7: Classification process of ECG signals with the trained Support Vector Machines.

Figure 6: Approach of Genetic Algorithm-Support Vector Machine

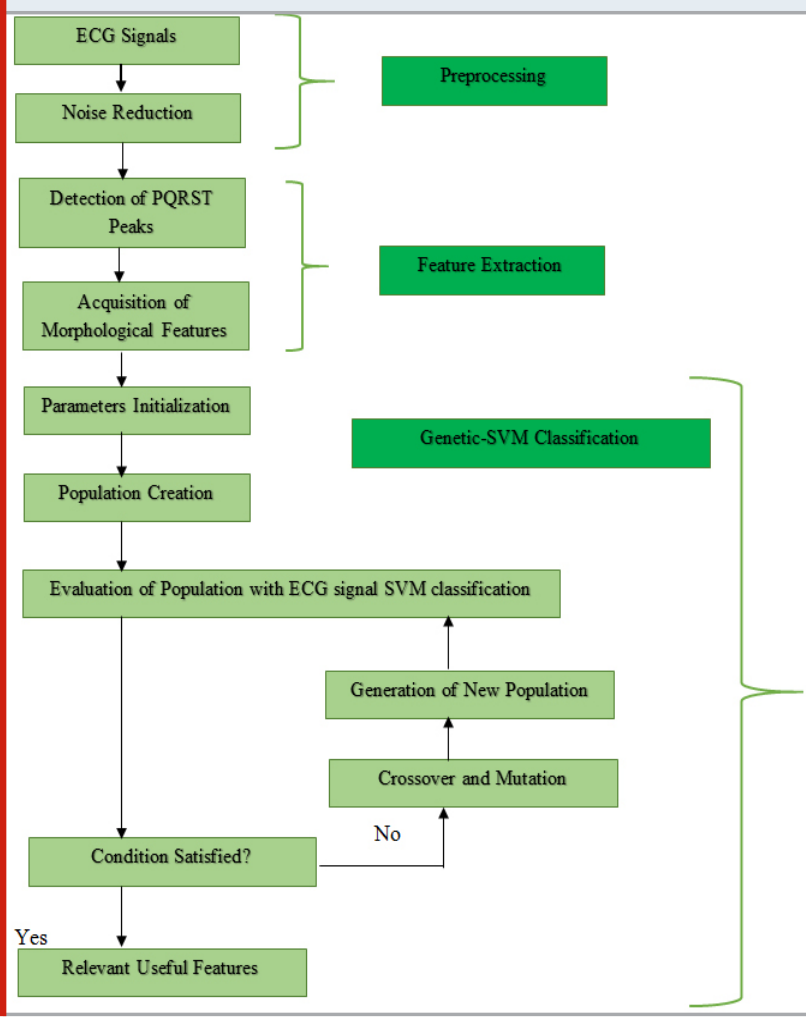

Table 4. Classification of ECG Arrhythmia using SVM with Linear and Polynomial Kernel

\begin{tabular}{|l|l|l|l|l|l|l|l|}
\hline S No & P, LR & P, LL & P, N & LR, LL & LR, N & LL, N & Overall \\
\hline 1 & 97.96 & 99.99 & 51.25 & 99.45 & 55.56 & 68.74 & 78.83 \\
\hline 2 & 92.63 & 98.21 & 54.26 & 99.45 & 53.24 & 68.74 & 77.76 \\
\hline
\end{tabular}

\section{RESULTS AND DISCUSSION}

For evaluating the proposed technique, more than seventy five percent of informational data which has
Table 5. Classification of ECG Arrhythmia using PCA_SVM with Linear and Polynomial Kernel

\begin{tabular}{|l|c|c|c|c|c|c|c|}
\hline S No & P, LR & P, LL & P, N & LR, LL & LR, N & LL, N & Overall \\
\hline 1 & 98.24 & 98.65 & 54.72 & 99.54 & 55.68 & 66.82 & 78.94 \\
\hline 2 & 99.32 & 99.45 & 55.45 & 97.28 & 55.68 & 66.82 & 79 \\
\hline
\end{tabular}

Table 6. Classification of ECG Arrhythmia using GA_SVM with Linear and Polynomial Kernel

\begin{tabular}{|l|l|l|l|l|l|l|l|}
\hline S No & P, LR & P, LL & P, N & LR, LL & LR, N & LL, N & Overall \\
\hline 1 & 96.26 & 98.45 & 99.26 & 99.99 & 89.75 & 98.11 & 96.97 \\
\hline 2 & 96.26 & 90.22 & 86.15 & 99.99 & 74.62 & 95.41 & 90.44 \\
\hline
\end{tabular}

been acquired from MIT-BIH arrhythmia database are being utilized to train the system of composition and the remaining are utilized for making the evaluation. In the initial state of experiment, the stage of selection is being omitted and the application of Support Vector Machine Classifier is being directly made to the entire space of original features. The results over the experimentation has been represented in table 4, 5 and 6 .

Figure 7: Performance evaluation of linear kernels with three different classification algorithms

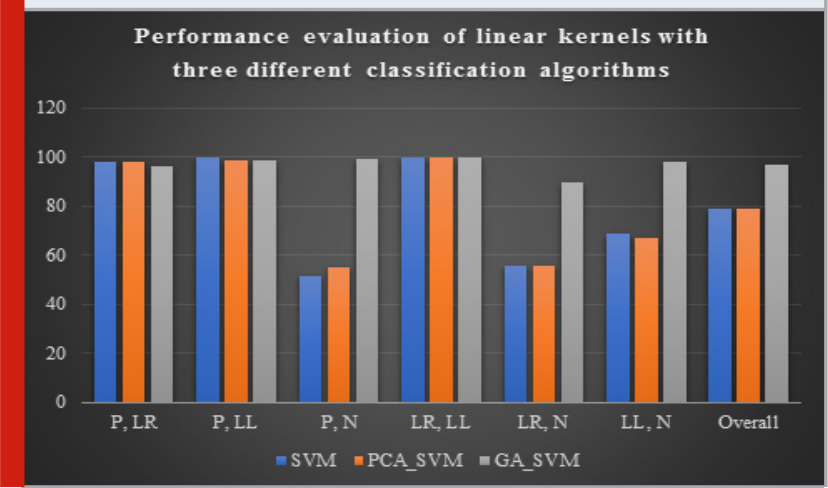

Figure 8: Performance evaluation of Polynomial kernels with three different classification algorithms

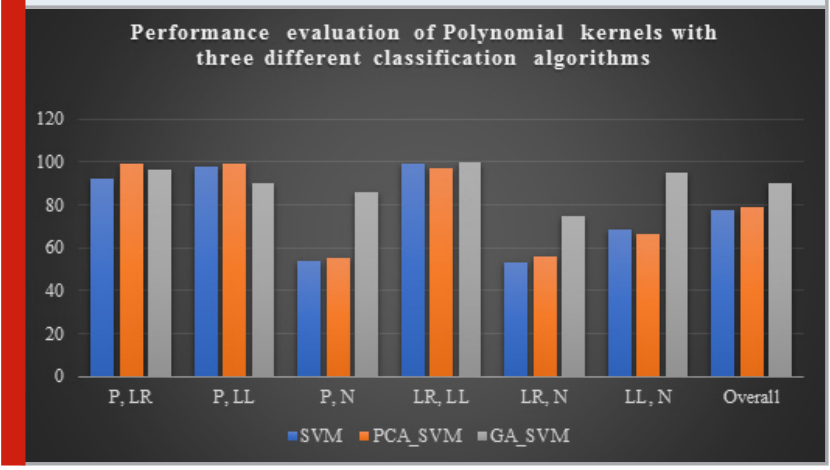


Figure 9: Evaluation between the fitness value and the population generation

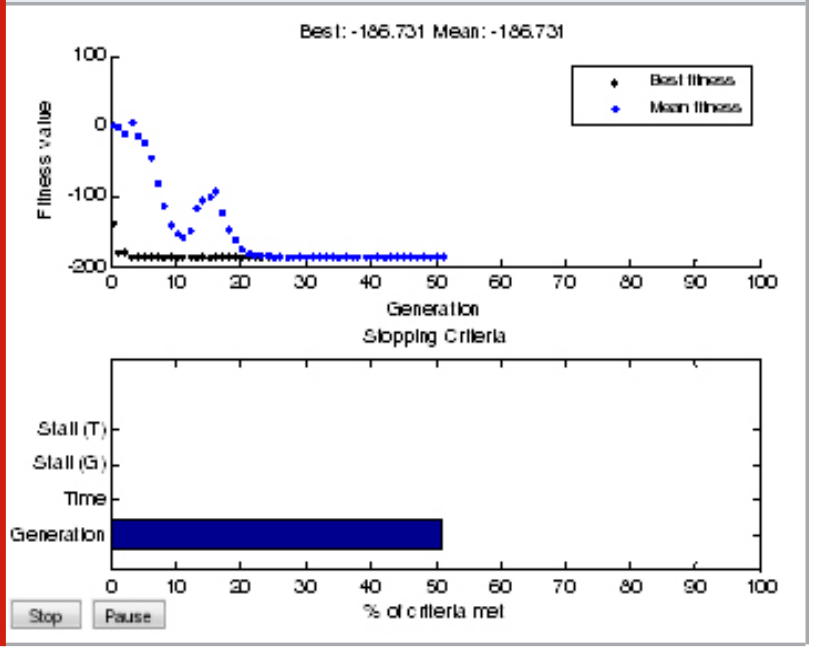

In the next part of experimentation, the proposed Genetic Algorithm-Support Vector Machine Classifier system is being utilized to the best feature subset which could optimize the classifier of SVM. The conclusion is made in such a way that the GA-SVM possessed with linear kernel generally performs well and it has been provided with $96.97 \%$ of classification accuracy in diagnosing the cardiac arrhythmias. Figure 7 shows the performance evaluation of linear kernels with three different classification algorithms and Figure 8 shows the performance evaluation of Polynomial kernels with three different classification algorithms. The simulation window which determines the evaluation between the fitness value and the generation has been depicted in figure 9.

\section{CONCLUSION AND FUTURE WORK}

In this proposed study, the proposal of a novel technique on the basis of Genetic Algorithm-Support Vector Machine for the selection of features and the classification of ECG signals has been made. The results over the experimentation has proved that the methodology for the right selection of features enhances the classification accuracy and quality. This happens and exists since few features might act as noise component, and degrades the classification accuracy. A simple and efficient GA-SVM technique that has employed for ECG classification is being presented with the datasets that has been acquired from MIT-BIH arrhythmia database. The future work includes the enhancement in deep learning techniques for classification by the determination of feature selection strategies.

\section{REFERENCES}

Acharya U R, et al., (2008). Automatic identification of cardiac health using modeling techniques: a comparative study. Inform. Sci. 178 Pages 4571-4582.

Acharya, U.R., Fujita, H., Lih, O.S., Hagiwara, Y., Tan, J.H., Adam, M., (2017). Automated detection of arrhythmias using different intervals of tachycardia ECG segments with convolutional neural network. Inform. Sci. 405 Pages 81-90.

Ahmad, A.M., Khan, G.M., Mahmud, S.A., (2013). Classification of Arrhythmia Types Using Cartesian Genetic Programming Evolved Artificial Neural Networks. In: Iliadis L., Papadopoulos H., Jayne C. (Eds.,) Engineering Applications of Neural Networks. EANN 2013. Communications in Computer and Information Science, vol. 383, Springer, Berlin, Heidelberg.

Bandyopadhyay, S, S.K. Pal, (2007). Classification and Learning Using Genetic Algorithms, Springer-Verlag Berlin Heidelberg.

Banerjee, S., Mitra, M., (2014). Application of cross wavelet transform for ECG pattern analysis and classification. IEEE Trans. Instrum. Meas. 63 (2) Pages 326-333.

Chazal, P., M. O’Dwyer, R.B. Reilly, (2004). Automatic classification of heartbeats using ECG morphology and heartbeat interval features, IEEE Trans. Biomed. Eng. 51 Pages 1196-1206.

Chang, C.C., Lin, C.J. (2011). LIBSVM: a library for support vector machines. ACM

Transactions on Intelligent Systems and Technology 2 Pages 27:1-27:27.

Clifford, G.D., F. Azuaje, P.E. McShary, (2006). Advanced Methods and Tools for ECG Data Analysis, Artech House, Norwood, MA.

Daubechies, I., (1998). Orthonormal bases of compactly wavelets. Commun Pure Appl Math 41 Pages 909996.

Ebrahimzadeh, A. Khazaee, (2010). Detection of premature ventricular contractions using MLP neural networks: a comparative study, Measurement 43 Pages 103-112.

Homaeinezhad, M.R., Tavakkoli1, E., Ghaffari1, A, (2011). Discrete Wavelet-based Fuzzy Network Architecture for ECG Rhythm-Type Recognition: Feature Extraction and Clustering Oriented Tuning of Fuzzy Inference System. In: International Journal of Signal Processing, Image Processing and Pattern Recognition, vol.4, no. 3, September.

Hsu, C.W., C.-J. Lin, (2002). A comparison of methods for multiclass support vector machines," IEEE Trans. Neural Netw. 13(2) Pages 415-425.

Kressel, U.H.-G, (1999). Pairwise classification and support vector machines. In: Advances

in Kernel Methods, Pages 255-268. MIT Press, Cambridge. 
Liu, T., Si, Y., Wen, D., Zang, M., Lang, L., (2016). Dictionary learning for VQ feature extraction in ECG beats classification. Expert Syst. Appl. 53 Pages 129-137.

Mark, R.G., G.B. Moody, MIT-BIH Arrhythmia Database 1997 [Online]. Available: http://ecg.mit.edu/dbinfo. html.

Martis, R.J., Acharya, U.R., Lim, C.M., Suric, J.S., (2013b). Characterization of ECG beats from cardiac arrhythmia using discrete cosine transform in PCA framework. Knowl.-Based Syst. 45 Pages 76-82.

Mitra, M., Samanta, R.K., (2013). Cardiac arrhythmia classification using neural networks with selected features. Proced. Technol. 10 Pages 76-84.

Osowski, S, T.H. Linh, (2001) ECG beat recognition using fuzzy hybrid neural network, IEEE Trans. Biomed. Eng. 48 Pages 1265-1271.

Takeuchi, K., Collier, N., (2003). Bio-Medical Entity Extraction using Support Vector Machines. Proceedings of the ACL 2003 Workshop on Natural Language Processing in Biomedicine, Pages 57-64.

Thomas, M., Das, M.K., Ari, S., (2015). Automatic ECG arrhythmia classification using dual tree complex wavelet based features. Int. J. Electron. Commun. 69 (4) Pages 715-721.

Vaseghi, S.V., (2008). Advanced Digital Signal Processing and Noise Reduction, 4th edition, John Wiley \& Sons. Wang, T.-Y., Chiang, H.-M., (2007). Fuzzy support vector machine for multi-class text categorization. Information Process and Management, 43 Pages 914-929. 